\title{
Evolution of Collective Motion in Light Polonium Nuclei
}

J. A. Cizewski, ${ }^{1,2}$ K. Y. Ding, ${ }^{1}$ N. Fotiades, ${ }^{1}$ D. P. McNabb, ${ }^{1}$ W. Younes, ${ }^{1,3}$ R. Julin, ${ }^{4}$ M. Leino, ${ }^{4}$ J. Cocks, ${ }^{4}$ P. Greenlees ${ }^{4}$ K. Helariutta, ${ }^{4}$ P. Jones, ${ }^{4}$ S. Juutinen, ${ }^{4}$ A. Kankaanpääa, ${ }^{4}$ H. Kettunen ${ }^{4}{ }^{4}$ P. Kuusiniemi, ${ }^{4}$ M. Muikku, ${ }^{4}$ P. Rahkila, ${ }^{4}$ A. Savelius, ${ }^{4}$ C. N. Davids,${ }^{2}$ R. V. F. Janssens, ${ }^{2}$ D. Seweryniak, ${ }^{2}$ M. P. Carpenter, ${ }^{2}$ H. Amro, ${ }^{2}$ P. Decrock, ${ }^{2}$ P. Reiter,${ }^{2}$ D. Nisius, ${ }^{2}$ L. T. Brown, ${ }^{2} \mathbf{O}$ S. Fischer, ${ }^{2}$ T. Lauritsen, ${ }^{2}$ J. Wauters, ${ }^{5}$ C. R. Bingham, ${ }^{5}$ M. Huyse, ${ }^{6}$ A. Andreyer

${ }^{1}$ Department of Physics and Astronomy, Rutgers University, New Brunswick, NJ 08903

${ }^{2}$ Physics Division, Argonne National Laboratory, Argonne, IL 60439

${ }^{3} N$-Division, Lawrence Livermore National Laboratory, Livermore, CA 94550

${ }^{4}$ Department of Physics, University of Jyväskylä, Jyväskylä, Finland

${ }^{5}$ Department of Physics and Astronomy, University of Tennessee, Knoxville, TN 37996

$\therefore \quad{ }^{6}$ Instituut voor Kern- en Stralingsfysica, K.U. Leuven, Leuven, Belgium

\begin{abstract}
The $\gamma$-ray spectroscopy of even- and odd-mass isotopes of polonium have been studied using arrays of Ge detectors coupled to recoil-mass analyzers, including recoil-decay tagging techniques. The level energies and $B(E 2)$ branching ratios can be reproduced by theoretical frameworks which do not explicitly include proton particlehole excitations across the $\mathrm{Z}=82$ shell, conclusions in contrast to those deduced from alpha-decay measurements.
\end{abstract}

The nuclei in the lead region exhibit a wide variety of shapes as a function of neutron and proton number, as well as excitation energy and angular momenta. As the numbers of valence protons and neutrons increase, the structure evolves from shell model behavior near $N=126$ to moderate collective oblate shapes. As the middle of the $\mathrm{N}=82-126$ neutron shell is approached, proton particle-hole excitations across the $\mathrm{Z}=82$ shell gap give rise to strongly collective oblate excitations, which coexist with the more moderate ground-state shapes [1].

In recent years, the structure of the lightest polonium isotopes has been probed using recoil separators coupled to arrays of Compton-suppressed Ge detectors. The use of recoil separators was critical to identify gamma rays associated with the evaporation residues, which are populated with cross sections considerably less than the dominant fission channel. For the lightest isotopes, the recoil decay tagging technique was used to identify prompt transitions in specific alpha-decaying isomers of the evaporation residues. Such studies have been completed on ${ }^{192-197}$ Po [2-5], and in the case of the odd-A isotopes, transitions associated with both $13 / 2^{+}$and $3 / 2^{-}$isomers were identified.

More recently, high-spin excitations were probed in ${ }^{198} \mathrm{Po}$ using the ${ }^{172} \mathrm{Yb}\left({ }^{28} \mathrm{Si}, 4 \mathrm{n}\right)$ reaction at $143 \mathrm{MeV}$ using the Jurosphere array of Compton-suppressed Ge detectors coupled to the gas-filled recoil separator RITU at the University of Jyväskylä. 


\section{DISCLAIMER}

This report was prepared as an account of work sponsored by an agency of the United States Government. Neither the United States Government nor any agency thereof, nor any of their employees, make any warranty, express or implied, or assumes any legal liability or responsibility for the accuracy, completeness, or usefulness of any information, apparatus, product, or process disclosed, or represents that its use would not infringe privately owned rights. Reference herein to any specific commercial product, process, or service by trade name, trademark, manufacturer, or otherwise does not necessarily constitute or imply its endorsement, recommendation, or favoring by the United States Government or any agency thereof. The views and opinions of authors expressed herein do not necessarily state or reflect those of the United States Government or any agency thereof. 


\section{DISCLAIMER}

Portions of this document may be illegible in electronic image products. Images are produced from the best available original document. 
TABLE 1. B(E2) branching ratios for non-yrast states in ${ }^{196} \mathrm{Po}$.

\begin{tabular}{lccccc}
\hline \multicolumn{1}{c}{ Transition } & \multicolumn{5}{c}{ B(E2) ratios } \\
& Delayed & Prompt & Vibrator & Rotor & $4 p-2 h$ \\
\hline $4_{2}^{+} \rightarrow 2_{2}^{+} / 4_{2}^{+} \rightarrow 4_{1}^{+}$ & $1.41(27)$ & $1.42(23)$ & 1.10 & $>20$ & $>20$ \\
$2_{2}^{+} \rightarrow 2_{1}^{+} / 2_{2}^{+} \rightarrow 0_{1}^{+}$ & $20.9(46)$ & $21.5(58)$ & $\infty$ & $2.04(K=0)$ & Large $E 0$ \\
\hline
\end{tabular}

In addition to recording prompt $\gamma$ rays at the target position in coincidence with recoils at the RITU focal plane, transitions associated with the decay of long-lived isomers, $t_{1 / 2} \approx 1 \mu s$, were measured with a single Ge detector behind the focal plane. The level spectrum deduced for the decay of the 856(17)ns $11^{-}$isomer in ${ }^{196} \mathrm{Po}$ is displayed in Fig. 1. These measurements essentially confirm the previous results [6] for the decay of this high-spin isomer, although additional non-yrast states have been observed following weak population in the isomer decay. The placements of the transitions, except those which directly depopulate the isomer, are confirmed by prompt $\gamma-\gamma$ coincidence measurements at the target position.

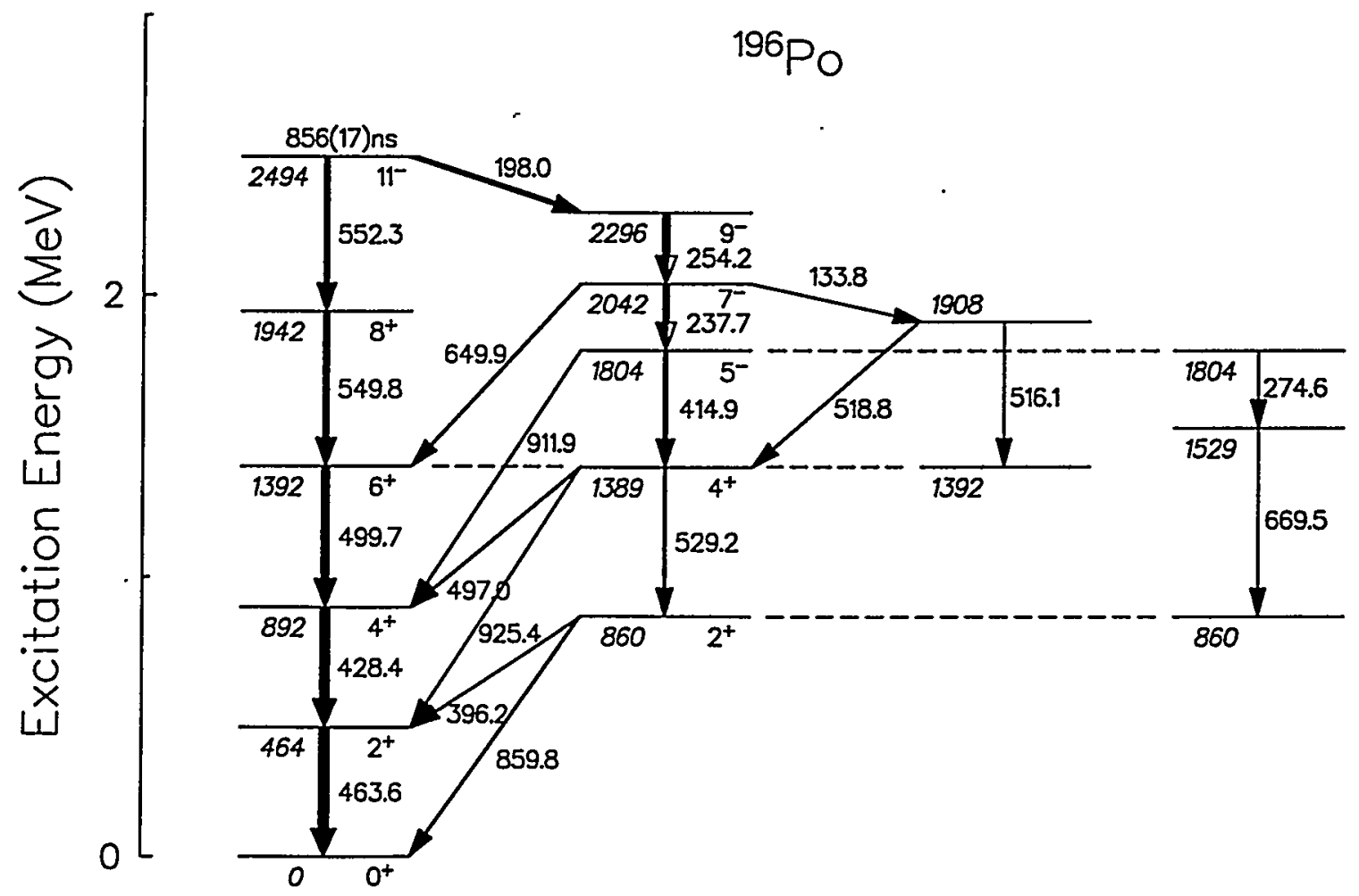

Fig. 1: Decay of $11^{-}$isomer in ${ }^{196} \mathrm{Po}$. 


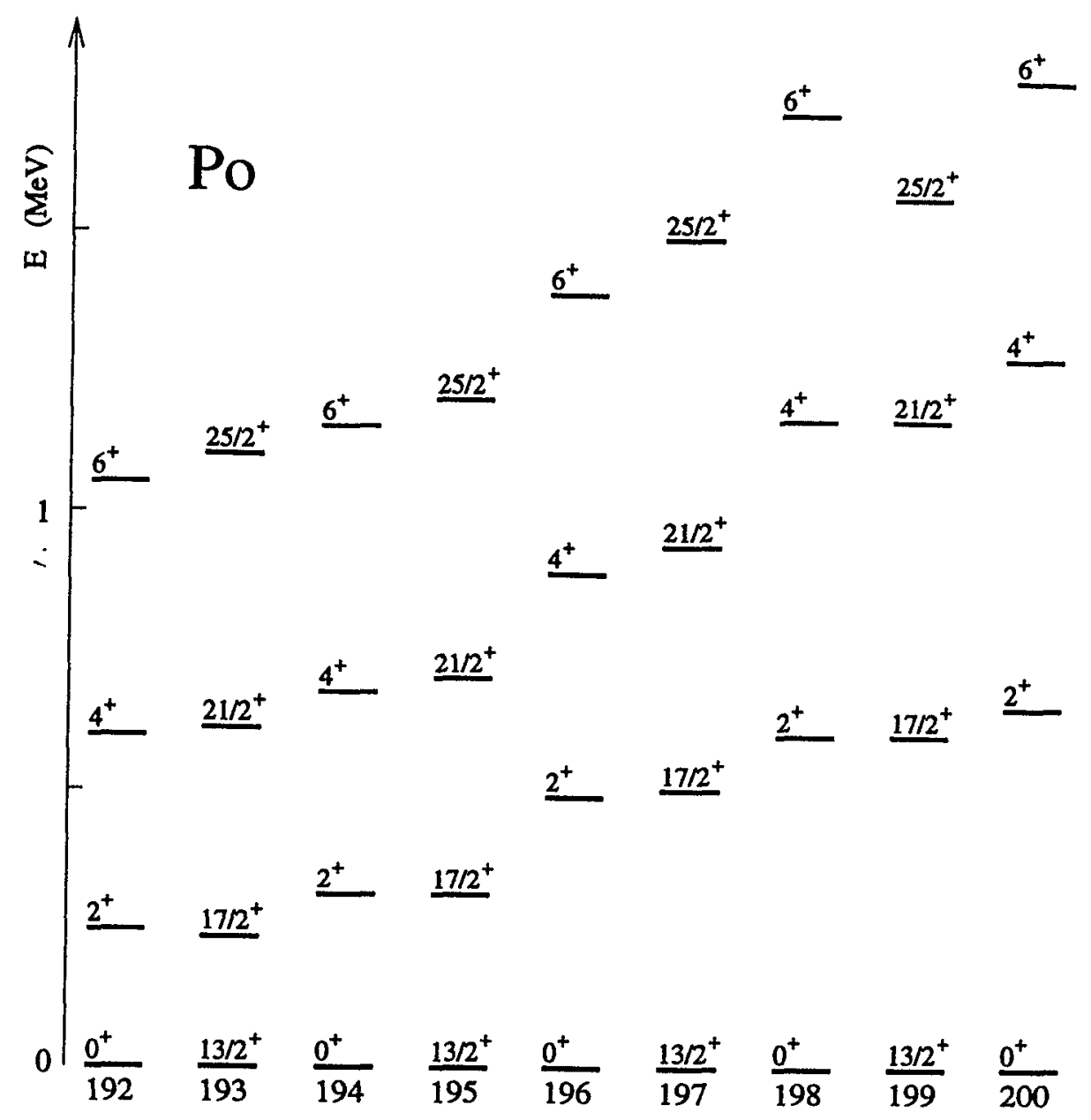

Fig. 2: Level systematics for ${ }^{192-200} \mathrm{Po}$.

In Table 1 the relative $B(E 2)$ branching ratios associated with the decay of the non-yrast states in ${ }^{198} \mathrm{Po}$ are summarized. The data from both delayed measurements and prompt spectroscopy gated on the 414-keV transition are tabulated; pure E2 transitions are assumed. These experimental results are compared with predictions for vibrational and rotational models, as well as expectations for 4-particle, 2-hole (4p-2h) proton excitations across the $\mathrm{Z}=82$ shell gap. For "intruder" $4 \mathrm{p}-2 \mathrm{~h}$ excitations, the non-yrast positive-parity states are candidates for the quasiband built on the "intruder" configuration, and allowed, intra-band transitions should be a factor of 20-50 times larger than forbidden, inter-band transitions. The experimental results are only consistent with the expectations of the vibrational model. In addition, the imbalance in $\gamma$-ray intensity required for the E0 transitions expected to characterize the $\Delta \mathrm{J}=0$ transitions between "normal" 2-particle, and "intruder" $4 \mathrm{p}-2 \mathrm{~h}$ configurations is not observed. The present results confirm those of ref. 7 , but are in marked disagreement with those of ref. 6 , which reported intensity imbal- 
ances. The level spacings, with nearly degenerate members of the 2- and 3-phonon multiplets, and the relative $B(E 2)$ branching ratios support a vibrational structure for ${ }^{196} \mathrm{Po}$. Detailed spectroscopy of the lighter isotopes, in particular ${ }^{194} \mathrm{Po}$, would further probe the structure of the Po nuclei and the role of $4 \mathrm{p}-2 \mathrm{~h}$ intruder configurations in the yrast and non-yrast states. A new analysis of ${ }^{194} \mathrm{Po}$ is in progress [8].

The low-spin levels of the ${ }^{200-192}$ Po isotopes, including the levels above the $13 / 2^{+}$ isomers in the odd-A nuclei, are summarized in Fig. 2. The gamma-ray data suggest that the lightest Po isotopes evolve from a harmonic vibrational structure in ${ }^{196} \mathrm{Po}$ to a more collective, anharmonic vibrator in ${ }^{192} \mathrm{Po}$. The structure of the excitations built on the $13 / 2^{+}$isomers in the ${ }^{193,195,197}$ Po isotopes, which follow a simple weakcoupling pattern, support this interpretation. If the yrast states in the core were complicated admixtures of "normal" and 4p-2h "intruder" configurations, which changed as a function of neutron number, the level spacings above the $13 / 2^{+}$isomers would not be expected to follow those of the core.

The evolution of low-lying structure from ${ }^{208} \mathrm{Po}$ to ${ }^{192} \mathrm{Po}$ has been successfully modeled in the particle-core coupling framework and the microscopic composition of the $2^{+}$states determined from quasiparticle RPA calculations [9]. The theoretical analyses suggest that it is the opening of the $\nu i_{13 / 2}$ shell in the lighter isotopes, and the attractive interaction between $i_{13 / 2}$ neutrons and the valence $h_{9 / 2}$ protons, which result in increased collectivity, which remains essentially of anharmonic vibrational character, in the lighter isotopes.

These conclusions are in contrast to results from the alpha decay of these Po nuclei, where the fine structure suggests that proton particle-hole excitations are a dominant component in the ${ }^{192}$ Po ground state [10].

This work is supported in part by the U.S. National Science Foundation and Department of Energy.

\section{REFERENCES}

[1] J. L. Wood et al., Phys. Rep. 215, 101 (1992).

[2] W. Younes et al., Phys. Rev. C 52, R1723 (1995)

[3] K. Helariutta et al., Phys. Rev. C 54, R2799 (1996).

[4] N. Fotiades et al., Phys. Rev. C 55, 1724 (1997).

[5] N. Fotiades et al., Phys. Rev. C 56, 723 (1997).

[6] D. Alber et al., Z. Phys. A 339, 225 (1991).

[7] L. A. Bernstein et al., Phys. Rev. C 52, 621 (1995).

[8] K. Helariutta et al., to be published.

[9] W. Younes and J. A. Cizewski, Phys. Rev. C 55, 1218 (1997).

[10] N. Bijnens et al., Z. Phys. A 356, 3 (1996). 